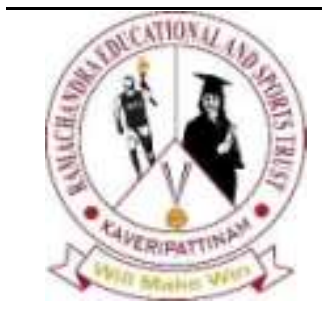

REST Journal on Emerging Trends in Modelling and Manufacturing

Vol: 7(4), 2021

REST Publisher

ISSN: $\mathbf{2 4 5 5}-4537$

Website: www.restpublisher.com/journals/jemm

\title{
A Review on Structural Equation Modeling and Its Classification
}

\author{
Vikrant Sharma, M. Ramachandran, Sathiyaraj Chinnasamy, Vimala Saravanan \\ Mechanical Engg. Department, Mody University, Rajasthan, India \\ REST Labs, Kveripattinam, Krishnagiri, TamilNadu, India. \\ Email: vikrantrsharma@yahoo.com
}

\begin{abstract}
Structural equation modeling is many dimensions are a statistic is the technique of analysis, which is structural Used to analyze relationships. This technique includes factor analysis and multiple regression analysis and Is a combination of measured variables hidden constructions. Structural equations specify how the set of variables are interrelated based on linear equations, cause and effect (cause models) or paths through statistically (path analysis) sorted networks. Structural Equation Modeling (SEM) is a quantitative research technique that integrates standard methods. SEM is often used for research, rather than to explore or explain an event a research study is designed to verify the design. Structural Equation Modeling (SEM) is standard A quantity that integrates methods Is the research technique. Used show causal relationships between SEM variables. The relationships shown in the SEM refer to the researchers' hypotheses. In general, these relationships cannot be statistically tested for diversion. Structural equation modeling is a small number of 'structures' Defined as a class of methods that represent the mechanisms, variations, and hypotheses of data that are inferred on the basis of parameters. 'Configuration' parameters. Path analysis is a special case of SEM. Most models you as seen in the literature, SEM are higher than path analytics. Between the two types of models the main difference is that all variables in the path analysis are measured without error Considers. SEM uses hidden variables to calculate the measurement error. Structural Equation Modeling (SEM) is a multiple regression Factor analysis and various techniques Integration is an advanced technique ANOVA. It evaluates the causal relationship between more than one dependent variable and several independent variables.
\end{abstract}

\section{Introduction}

Structural equation modeling is the label for the various Experimental and observational research by scientists in both methods used in science, business and other fields. It is social and behavioral widely used in science. Structural Equation Modeling (SEM) is a statistic used to create and test statistical models. Technically, they are mostly causal models. It is a hybrid technique that confirms factor analysis, path analysis and regression. Includes features, which are considered a special event of SEM. SEM promotes verification rather than modeling; therefore, it is more theoretical than the development of a theory suitable for experimentation. It usually starts with a hypothesis, refers to it as a model, the function analyzes the structure of interest with a measuring instrument and testing the model. The accepted theory, or SEM, refers to a verified model and the values of the free parameters. It can also be used as a stimulus by using data to evaluate. Often the initial hypothesis requires adjustment in light of sample evidence, but SEM is rarely used analysis. Path analysis, a precursor to structural equation modeling and subgroup, a method for detecting and evaluating the effects of a set of variables acting on a particular end through a number of causal pathways. Route analysis is a reason to examine connections within a defined network is the modeling approach. This method is structural equation modeling (SEM), Covarian's structural equation modeling (CSEM), also known as Covarians Structures Analysis or Covarians Structural Analysis. To perform the path analysis, write the names of the variables in the squares and attach the squares to the arrows. It refers to the effect of one on the other as well as regression. Path analysis works in two ways; before and after running the regression. Confirmation Factor analysis (CFA) is a statistical technique used to check the factor structure of a set of observed variables. Observed variables and their underlying implications Allows the CFA researcher to test the hypothesis that there is a relationship between structures. For example, there are two factors for calculating cooperation in measurements and these factors Even if they are said to be unrelated, the researcher can develop a model that controls the relationship between factor A and factor B to zero.Therefore, affirmative factor analysis focuses on the implementation of assumed networks as a whole, improves statistical power by modeling measurement error, and strengthens statistics. Basically provides a theory based approach to data reduction. Confirmation factor analysis (CFA) is the basic first step in implementing most types of SEM models. You must first do this to verify the measurement quality of all the hidden constructions you use in your structural equation model. A meta-analysis is a statistical analysis that integrates the results of several scientific studies. Meta-analysis can be done when there are multiple scientific studies that solve the same question, and each individual study measurement is expected to be somewhat erroneous. Meta-analysis is a statistical process for combining data from multiple studies. This general effect occurs in a study of therapeutic effect (or effect size) When consistent from the next study, identify you can use Metaanalysis to view.Meta-analyzes help to establish statistical significance throughout the studies, otherwise they may have conflicting results. This is important because the statistical significance increases the validity of the observed differences. 
This increases the reliability of the information. The model test is a complete test for a model that has not been tested before. In this case the technical specifications and the requirements of the model will be considered in detail. If there are multiple devices in the equipment that affect the specifications, each component will be tested. In machine learning, model testing is referred to as the process of evaluating the performance of a fully trained model in a set of tests. There are several statistical measurements used to evaluate test results, including average square errors and receiver operating characteristics curves.

\section{Structural Equation Modeling}

Structural equation modeling is the label for the various methods used by scientists in both experimental and observational research in science, business and other fields. It is widely used in social and behavioral sciences. [1] Importantly, ANEVA and SEM are the most familiar techniques of regression. These basics apply equally; However, I recommend that researchers do data management tasks outside of their dedicated SEM software packages. Bootstrapping captures the range of potential applications in SEM, but if modified or "robust" bugs are fixed it goes beyond the maximum possible rating what benefits does it offer? Not clear. Their chapter will be useful for new and experienced readers, taking the time to discuss reporting practices for SEM topics that do not currently have detailed guidelines.

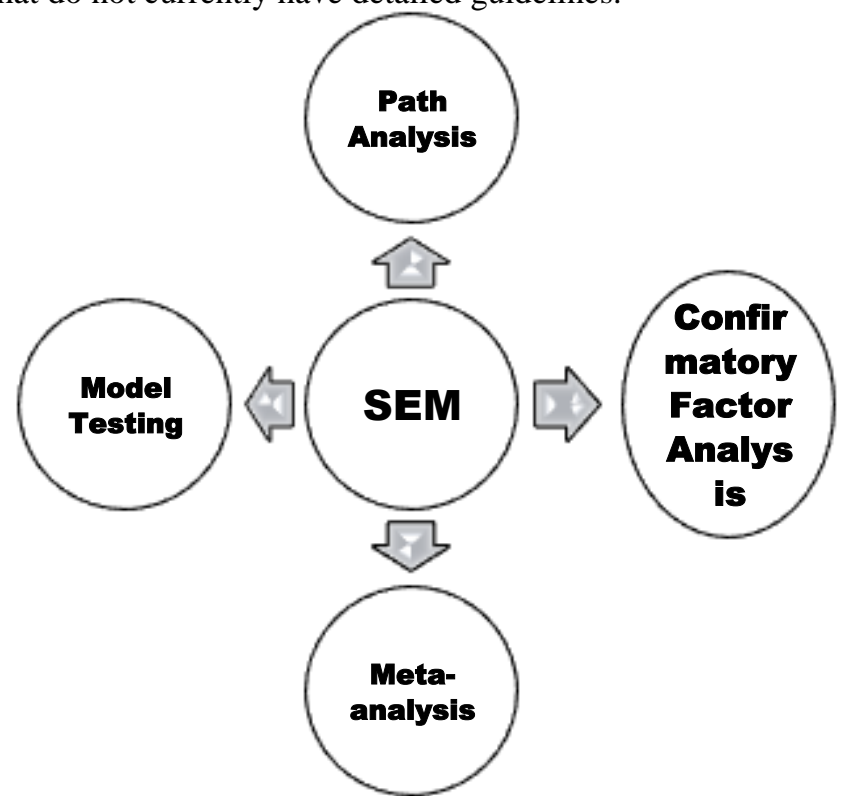

[2] The origin and development of SEM has been considered an important statistical development in the social sciences in recent decades, and this "second generation" multidisciplinary analysis method is widely used in theoretical studies and empirical validation in many fields. In addition, SEM can also provide group comparisons with a complete model, resulting in clearer records than traditional ANOVA. Therefore, this review is comprehensive for standard SEM applications to solve problems in construction delivery is the search. Therefore, construction management SEM designs have the potential to enrich themselves by considering time delays. An SEM cross-sectional study is the study of variables and the structure of a structure over a period of time, as well as over time includes a lengthy study between constructions over time about connections. There are two main classifications of (hidden) variables in SEM. [3] the first two of the six chapters covering this area are generally historical events related to the evolution of the SEM and specifically related to the LISREL project. The last two articles in this section take a psychometric perspective and explore aspects of reliability in SEM. The five chapters covering the last part of the book describe the applications of some recent research areas in SEM. This chapter is not for neophytes of contact models; it is non-linear Experienced SEM researchers working with models should be of considerable interest. The final two chapters will fully explore the different aspects of SEM based on typically measured categorical data. In fact, this article came from SEM, which is within the framework Seems to stimulate growing interest in the IRT modeling area within. This book should be of considerable interest and value to all researchers who wish to conduct statistically superior applications of SEM,despite their level of expertise. [4] In my opinion, the balance between a discussion of this issue and empirical treatment is the right one to obtain sound endorsements from congressmen, academics, think tanks, and lawyers. If I have ever taught a graduate seminar at SEM, I should read this article. In other episodes, Hartman discusses bootstrap methods in SEM, while Steiger discusses communication methods across different models. Presents methods for comparison, Browne and Nesselroade discuss long issues at SEM. Discuss, Goldstein and Brown Discuss multilevel analysis. The scope of the SEM section is very large and contains more chapters than any other section, this section it is a reflection of both the recent popularity and the extent and scope of Professor McDonald's contributions to this category. [5] SEM our analysis shows that as a final feature off's previous applications, 85 percent of the authors did the analysis using LISREL. In contrast to the previous section the focus was on monitoring the number of articles using SEM over time, the model given by the analysis unit in this section. In 81 cases, SEM was used only to examine the measurement structure based on the set of observed variables. Single-indicator structures are geeky because they ignore the reliability of the measurement, which is one of the problems SEM specifically designed to avoid. The primary goal of a researcher should be to obtain high-quality data so that in some cases SEM may not be an appropriate method. Of SEM our own assessment of model identification in previous applications suggests that most models were actually theoretically identified. One of the 
most common mistakes in using SEMs is to have little or no focus on source data and to quickly calculate a correlation matrix and rush to sample evaluation and testing. [6] This model is called mathematical "normal distribution". In fact, used in SEM Most statistical procedures are inherent in the distribution of observations in their derivatives involves the assumption of character. To test the hypothesis of the statistical significance of sample parameter estimates,by a significant ratio, the z-score is very important in SEM. This SEM software enables intensive external detection, which may cause deviations from the default. In the event of a more serious violation, SEM users have several options. They can be divided into three types. It turns out that the modeling strategy with SEM users is increasingly successful. The use of subsequent temporary changes in the modeling approach creates a fundamental problem with the stability of the SEM. The availability of software with highly user-friendly interfaces is not specific to SEM. However, Lavanin is still in its infancy with JASP through SEM (Structure Equation Modeling) module integration, so its application may be temporarily difficult. [7] Traditional monolithic SEM programs offer only one evaluation solution. This feature shifts the importance of specializing in a particular SEM project to specializing in SEM. Despite the growing popularity of SEMs in the social sciences, the complexity and variability of script-based model definitions Variation of commands between programs may have limited the use of SEMs among less sophisticated computer users. [8] The basic facts keep coming back again and again. The traditional chi-square goodness of the test fitted in the SEM is the acceptance-support test of the statistically zero hypothesis of the correct model fit, i.e., the model excludes zero from the data. Accepting H0 supports the model. This acceptance-support test of zero conflict (AS0) has little direct value for a number of reasons. First, the perfect fit this "stand hypothesis" is irrelevant because SEM models are highly constrained and have zero potential for precision matching. PB indicates that the SEM fit codes index model does not fit incorrectly, as do the mismatch measurements in other types of analyzes. There are at least two basic reasons for this. First, SEM compliance codes minimize many inconsistencies. When SEM is used in more traditional areas such as ANOVA and MANOVA, simple hypothesis tests rule out the decision-making process, and PB confirms that "no one is talking about approximate bias or approximate mean difference" in such fields. "The publication of SEM analyzes based on less than 200 samples should be completely ruled out." [9] However, the hypotheses of SEM models are technically complex and could be understood by some researchers because their work did not arouse the interest of psychologists surrounding SEM strategy at the time. Multilevel For hidden variables SEM regression Modeling (ML-SEMs) is another advancement that has been adapted to multi-level analysis (MLM). He also provided a way to evaluate SEM available Using software two-level SEM. The first assumption made in the literature in the context of combining SEM with MA is to fit the structural equation model into the meta-analyzed Covariance or correlation matrix. In general, the golden rule for MG-SEM analysis is that the hypothesis of the hypothetical model is significant at 0.05 Otherwise, the C-squaredifference statistic using the researcher can go to the next level. In the dynamic development of SEM, there were also statistical and philosophical controversies over the use of this analytical technique. [10] Our review focuses on the empirical applications of SEM: (1) CFA models, calibration or verification research; (2) PA models (if evaluated using software that allows latent variable modeling); And (3) measurement and configuration SEM models that combine both components. When using SEM, there are several important issues in evaluating the measurement model or testing the suitability of individual or simultaneous structural relationships. Another basic assumption for SEM That is, the theoretical relationships considered in the experimental models represent real relationships in the population. being studied.

\section{Path Analysis}

Path analysis, a precursor and subgroup of structural equation modeling, is a method for detecting and evaluating the effects of a set of variables acting on a given end through a number of causal pathways. [11] Path analysis is an extension of multiple regressions, including multiple regression models or simultaneously estimated equations. Path analysis can be considered as a special case of SEM in which the structural relationships between the observed (negatively latent) variables are modeled. Therefore, path analysis (or more generally SEM) is sometimes referred to as causal modeling. [12] SEM is a general analytics framework that specializes in a wide variety of models. For example, multiple regression, path analysis, and confirmation factor analysis are special cases of the SEM model that have historically been widely used by behavioral scientists. Causal modeling with latent variables conceptually reflects the union of the latter two techniques by emphasizing the psychometric perspective characteristic of factor analysis and the causal modeling characteristic of path analysis. [13] Bootstrapping capabilities in SEM software are generally unknown and rarely used. SEM software considers many linear multivariate models, such as regression, simultaneous equation, path analysis, and canonical interaction, to be specialized in SEM. Take advantage of this capability in packages. [14] Early Contributions Worked in a company for decades in path analysis and factor analysis. In its current form, the SEM is a broad integration system that covers a wide family of statistical analyzes, including unique variables, latent class factors, and features of multi-level analytics. As a starting point, the reader will be familiar with the introductory concepts of latent variables and path analysis, which include path analysis, confirmation factor analysis, model identification, evaluation methods, modeling diagrams, and model fit. The effect of such a relative residual variance the path analysis can be illustrated with a simple example using distortion. [15] This includes path analysis (regression analysis), in which the equations representing the effect of one or more variables on another are resolved to evaluate their relationships, thus, for variables. Indicates the hypothetical causal relationship between Tested. The relationship between factor ( $\mathrm{s}$ ) and behavior and / or environmental characteristics is determined by path analysis. [16] The central part of the book consists of three chapters. In Chapter 1, Muller discusses linear regression and path analysis, introduces route maps and the Zoresk-Keesling-Wiley coding system, explains the degradation of combinations into direct, indirect, and total effects, and presents some issues in model identification. There are some typographical or major errors in 
the book. The two most notable is the path the reason for the analysis "Samuel" Wright and the "Digit" subcommand in EQS is credited with printing various matching codes instead of decimal places. In general, the product quality of the book is high. [17] Two methods are currently in use to specify SEM models in scripts. The first centers on specifying the parallel variations of the apparent and latent variables and the measurements that define the mean structure. The second method is based on route analysis and on the route map uses a small profile for paths and variables. First, we will use the path analysis method to specify the model. In this approach, first define the variables; we follow regression, variance and parallel paths we mention. While the path analysis method is preferred for some models, it is often easier or necessary to use measurements to specify a model. Many advanced models are available Open Mx do not have an equivalent path map, so their fusion must be specified via algebraic metrics. [18] Factor model and path model are two basic models within the structural equation modeling framework. Once a factor model is established, the analysis often goes a few steps further, for example, Absorbs and exposes problems by adding age-like predictive variables to read age differences in latent variables. Path analysis with graphical representations of effects and effect decay Comes from genetic research, where Wright (1920) proposed a method to predict the inheritance of the bivalve form of guinea pigs. [19] Any SEM program can analyze the path through the observed variables as a process, although most have more code (And the ability to write that code) are required, as the process will automatically generate multiple statistics. The process uses the regression of normal minimum squares to estimate the parameters of each equation, which is a common practice in observed variable path analysis.

\section{Confirmatory factor analysis}

Confirmation Factor analysis (CFA) is the process of checking the factor structure of a set of observed variables is a statistical technique used. For observed variables and their underlying implications The CFA allows the researcher to test the hypothesis that there is a connection between structures. [20] The definitive factor analysis provided a statistical test for the hypothesis, while most of the original studies relied on the interpretation of the zero-sequence correlations of the Campbell and Fiske (1959) criteria. The first criterion is sample size. Confirmation factor analysis is based on the large sample theory, viz. Results may be incorrect when the sample size is small. Once the database is created (or discovered) the researcher believes in the relationship between the functions in which the appropriate model should be developed. Confirmation factor analysis can estimate the reliability of the model through various benefit-fit indices. Data and basic model are analytical parameter estimates such as optimal stabilization factor. For a variety of reasons Wrong results may come out. Maximum probability confirmation factor analysis is an alternative that is often not used in clinical verification tasks. Confirmation factor analysis offers several unique benefits.[21] For me, The The CFA is best defined as a decision rule that accepts or rejects one or more hypotheses about population factor structure based on sample data. That is, CFA is about imaginary testing. Unfortunately, the techniques commonly used in CFA are what we keep in mind when using them Does not always match the hypotheses held. The debate over CFA and EFA is like arguing about the 'best' taste of ice cream: you pay the price and pick your choice. Take it. I would like to provide a brief simulation to illustrate some important differences and similarities between EFA and CFA. Second The technique is to use LISREL, which is commonly used in CFA. Therefore, for data and theory, CFA is a research practice in which the researcher collects data considering the relationships between them. However, 'grind' helps. There are some important differences between these two techniques. For example, EFA values more importance as indicators of EFA dimension, while CFA emphasizes good compatibility. [22] Discussion of CFA and SEM techniques should begin with the vocabulary and graphics commonly used in these types of articles. CFA is a is the stabilization technique, which is driven by theory. We like to think of SEM as CFA and many other setbacks because SEM is a validation technique, but it can also be used for research purposes. Rubric focuses on reporting non-technical CFA and SEMs to effectively communicate the full range of results and responsible application of the technique required to verify the validity of models and individual parameters. In other words, like the CFA, the researcher explores the importance of individual structural pathways that represent the impact of a latent structure or the latent structure in the observed variable. Finally, for the CFA, we would like to know the reliability of the observed variables in relation to latent constructions, i.e. Squared Multiple Correlations (SMC).[23] The CFA is the main statistical method for evaluating such a question. Therefore, it is important to understand the status of reporting practices in CFA applications within psychological research. To date, few reviews have examined specific reporting practices for the CFA. Three additional small studies have reviewed CFA reporting practices. In addition, although previous reviews have provided data about one sample per study, many CFA articles test and report on multiple samples. We report our review of CFA studies; we make recommendations for reporting guidelines, and provide a checklist above. An important aspect of CFA is the accuracy of the event under study, and it allows researchers to specify more complex hypotheses Allows. Another important thing is related to CFA studies. As mentioned above, the most common use of CFA is quantitative improvement and verification. The identified CFA articles were reserved for coding by approximately three authors. The CFA was used to study the remaining studies, constructions or theories (15.8\%) that did not involve instrument verification, or to evaluate the measurement sample before conducting the SEM (8.8\%). [24] Like other statistical methods, the CFA relies on assumptions. Apart from distribution issues, there is discussion about the sample size required to use CFA techniques. Although it is important to note that these guidelines are for research analysis and that the CFA coefficients reduce the measurement error. It is hoped that these criteria will provide an approximate guideline for CFA studies. With CFA, researchers use various matching codes to determine if sample data fit is acceptable. Articles that were difficult to pinpoint exactly how the CFA models were developed were given "weak theoretical support" ratings, and studies that could not determine the theoretical basis for creating the model (s) based on the information provided. Ratings of "small / no support" [25] although ML is the most common evaluation The method for CFA samples is rarely used to evaluate EFA samples. With the advent of advanced EFA methods, accidental matching of CFA models can be avoided. As discussed, a 
long-standing problem with FA utilization is the fact that CFAs are factor structures created by EFAs. Reflect. In order to demonstrate the differences between EFA and CFA, the CFA model could not be related to the data. Researchers mistakenly think that CFA is only used to verify or confirm hypotheses, but researchers often conclude that CFA Are studying. Use the method. In a final note, researchers should be aware that it is reasonable to follow the bad-fitting CFA model with EFA.

\section{Meta-analysis}

Meta-analysis is a statistical process that integrates the data of several studies. Treatment this is common when the effect (or effect scale) is consistent from one study to the next Meta-analysis can be used to identify the effect. [26] In a meta-analysis, the controversy over how the differences between the studies It should be taken into account that the magnitude of the estimated effect measurements remains unresolved. This research question indirectly considers the population of studies analyzed in the meta-analysis, and whether future studies should be conducted or previously unknown studies may emerge. Expects. Now look at the meta-analysis of $\mathrm{C}$ comparative studies, in which the answer variable is binary: improved / not improved or good response / poor response in clinical trials, or cross-sectional or chronic disease / epidemiology not in studies. For the sole reason that the magnitude of its effect is significantly different from the measurements of the first five studies, it is incorrect to exclude study 6 from the meta-analysis. [27] The nature of the exposure effects literature and the questions discussed in this review, meta-analysis of exposure relationship research has many advantages and none of these disadvantages. Although these studies are not included in the meta-analysis, they will be discussed later in this article. A meta-analysis of this review and expression Includes studies of unreinforced expression and expression published between 1968 and 1987. Many initial studies have found that there were no improper control groups or conditions used or any restrictions, so effect scale estimates could not be obtained. For Meta analysis. Finally, meta-analysis only includes studies that use human subjects. Hand-square the analysis ensured that both the distribution of pairs of system and object variables in each other's categories and the view of the frequency tables were distributed "approximately" based on the variables included in the Meta analysis. Within the courses. However, a meta-analysis of the effect of the type of effect on the magnitude of the exposure effect gives clear results. [28] Ours Although the sample of leadership courses we found for the meta-analysis were suitable for review by swimmers and colleagues, only 14 of the 56 documents we included in our review were included. In interpreting our meta-analysis, readers should keep in mind the research paradigms used by investigators to study the evaluation of female and male leaders. Based on studies that have examined the most frequently researched leadership styles, studies conducted at Early and Johnson companies have found that female and male leaders do not differ from each other in style or work-style. Differ in the course of leading democratically or arbitrarily. Therefore, the tendency of test subjects to devalue women in leadership roles, which produced an average weight effect level of 0.07 in our meta-analysis, was much weaker than trends such as discrimination against women in the hiring environment and physical depreciation Geeky people. [29] Studies examining the effects of certain conditions in one article In meta analysis Not included But will be reviewed elsewhere. Third, to add to the meta-analysis, a study author bias must have minimal control. The main purpose of metaanalysis is their effect is to explain the difference between the characteristics of the treatment with respect to the variability of the doses.

\section{Model Testing}

The model test is a complete test for a model that has not been tested before. In this case the technical specifications, the requirements of the model are detailed Will be considered. If there are multiple devices in the equipment that affect the specifications, each component will be tested; [30] the sample size issue discussed prior to Any method of parameter evaluation and sample testing performed according to the SEM method is in practice. For both analysis (e.g., measurement model development) and verification (e.g., testing of latent variable model), parameter evaluation and sample testing in SEM the use of identical subjects basically invalidates the statistical theory. Sample testing of the SEM system is usually (a) the probability ratio test statistic, also known as the C-Square Goodness-of-Fit Index; (b) optional describes the benefit-fit codes and (c) the criteria for obtaining the basic obtained parameter ratings and their standard errors. [31] Production prototype of high quality Software is rarely given high priority. In a research environment, code is usually written by scientists and is compatible (and working) with new programs, often with attached upgrades. It may be helpful to use the old code. Although such a process contributes to the efficient development of prototype models, software development the disconnected code of the process is generally difficult to read, unreliable and expensive to maintain. in the ongoing sample testing / development process. [32] These findings present significant obstacles to computational theory. A simple type of model that ignores subtle differences and distinguishes living objects from inanimate objects explains more variation than any other model tested, including a lower-temporal model based on intermediate-complex landscape features. [33] In this section, the research theoretical model tested in this study, we outline several ideological relationships derived from the literature used to construct the structure of organizational culture. The research model tested in this study raises the question of whether visible aspects of organizational culture affect the level of organizational performance. Proposed to solve. The proper use of the LISREL method is carefully considered in the context of the following guidelines to be considered. (i) the theoretical basis of the overall model to be tested, (ii) the validity of the measured variables, (iii) The nature of the relationship between the variables observed and the latent variables observed in the model, (iv) the directional cause, and (v) the errors in the measurement of the variables and the overall sample errors. [34] Therefore, limited sample verifications are not modified, but complement traditional sample testing techniques. Testing with sample validates presents the problem of creating a test case sample verification problem and interpreting the opposite examples as test cases. In most cases, the sample is literary with checkers Used for test reaction systems; it reads input values from sensors and sets output values accordingly. Sample 
checks can be used to automatically retrieve test packages that meet a given criterion. Model Checkers state explosion the size problem is very important when using sample checkers to create a test case as it is very susceptible to problem. Most of the work in testing with sample checkers is done using non-research prototypes been published to the public, some tools are generally available. [35] Partial sample testing should be considered more desirable than full sample testing. In general, the notion that uncertain formulas and parameter values should be established as independently as possible from the structure of the whole model is a fraction of the structure available for use in partial sample testing Experience shows that the allowable amount of data is small. The partial sample test does not guarantee the desired behavior of the whole sample without some problems in creation or parameter evaluation until the whole sample is simulated. Indicates that there may be. Therefore, it was found that some partial sample testing of a small part of the evaluation and reporting subsystem was necessary before the entire subsystem could be tested. Increasing the sequence of delays would make sense as the next phase of the partial sample test. But there is no need to argue in the present case that this behavior is based solely on high delay, third order.

\section{Conclusion}

Importantly, these criteria apply equally to SEM as the most familiar techniques of variance (ANOVA) and regression; however, the researchers found their own I recommend doing data management tasks outside of SEM software packages. Possible in bootstrapping to attract SEM in range Applications, but what benefits it offers is not clear from this chapter beyond the modified or maximum feasibility estimate. "robust" fixed bugs. SEM is a typical analytics framework that specializes in a wide variety of models. For example, multiple lag, path analysis and stabilization factor analysis are typical of the SEM model Special events are, historically, behavioral widely used by scientists. The affirmative for factor analysis hypothesis also provided statistical testing; Most of them Original studies of zero-sequence interactions by the Campbell and Fiske (1959) criteria relied on explanation. The first criterion is sample size. In a meta-analysis, the controversy over how to differentiate between studies in terms of the magnitude of the estimated effect scales remains unresolved. This research question presupposes that the population of studies the analysis in the meta-analysis is implicit and future studies may or may not have been previously known as emerging studies. High quality the product prototype of the software is rarely given high priority. In the research environment, the code is usually written by scientists, and in new programs that are compatible (and work with) improvements. Using the old code is very efficient. Although such a process contributes to the efficient development of prototype models, software development The disconnected code of the process is generally difficult to read, unreliable and expensive to maintain. the ongoing sample testing / development process.

\section{Reference}

1. Hoyle, Rick H., ed. Handbook of structural equation modeling. Guilford press, 2012.

2. Xiong, Bo, Martin Skitmore, and Bo Xia. "A critical review of structural equation modeling applications in construction research." Automation in construction 49 (2015): 59-70.

3. Byrne, Barbara M. "Structural equation modeling: Perspectives on the present and the future." International Journal of Testing 1, no. 3-4 (2001): 327-334.

4. Hancock, Gregory R., and Ralph O. Mueller, eds. Structural equation modeling: A second course. Iap, 2013.

5. Baumgartner, Hans, and Christian Homburg. "Applications of structural equation modeling in marketing and consumer research: A review." International journal of Research in Marketing 13, no. 2 (1996): 139-161.

6. Gana, Kamel, and Guillaume Broc. Structural equation modeling with lavaan. John Wiley \& Sons, 2019.

7. von Oertzen, Timo, Andreas M. Brandmaier, and Siny Tsang. "Structural equation modeling with $\Omega$ nyx." Structural Equation Modeling: A Multidisciplinary Journal 22, no. 1 (2015): 148-161.

8. Steiger, James H. "Understanding the limitations of global fit assessment in structural equation modeling." Personality and Individual differences 42, no. 5 (2007): 893-898.

9. Tarka, Piotr. "An overview of structural equation modeling: its beginnings, historical development, usefulness and controversies in the social sciences." Quality \& quantity 52, no. 1 (2018): 313-354.

10. Shah, Rachna, and Susan Meyer Goldstein. "Use of structural equation modeling in operations management research: Looking back and forward." Journal of Operations management 24, no. 2 (2006): 148-169.

11. Lei, Pui-Wa, and Qiong Wu. "Introduction to structural equation modeling: Issues and practical considerations." Educational Measurement: issues and practice 26, no. 3 (2007): 33-43.

12. Tomarken, Andrew J., and Niels G. Waller. "Structural equation modeling: Strengths, limitations, and misconceptions." Annu. Rev. Clin. Psychol. 1 (2005): 31-65.

13. Narayanan, Anantharam. "A review of eight software packages for structural equation modeling." The American Statistician 66, no. 2 (2012): 129-138.

14. Little, Todd D. Longitudinal structural equation modeling. Guilford press, 2013.

15. Beran, Tanya N., and Claudio Violato. "Structural equation modeling in medical research: a primer." BMC research notes 3, no. 1 (2010): 1-10.

16. Mueller, Ralph O. Basic principles of structural equation modeling: An introduction to LISREL and EQS. Springer Science \& Business Media, 1999.

17. Boker, Steven, Michael Neale, HermineMaes, Michael Wilde, Michael Spiegel, Timothy Brick, Jeffrey Spies et al. "OpenMx: an open source extended structural equation modeling framework." Psychometrika 76, no. 2 (2011): 306317. 
18. Jak, Suzanne. "Introduction to Meta-Analysis and Structural Equation Modeling." In Meta-Analytic Structural Equation Modelling, pp. 1-14. Springer, Cham, 2015.

19. Hayes, Andrew F., Amanda K. Montoya, and Nicholas J. Rockwood. "The analysis of mechanisms and their contingencies: PROCESS versus structural equation modeling." Australasian Marketing Journal 25, no. 1 (2017): 7681.

20. Cole, David A. "Utility of confirmatory factor analysis in test validation research." Journal of consulting and clinical psychology 55, no. 4 (1987): 584.

21. Hurley, Amy E., Terri A. Scandura, Chester A. Schriesheim, Michael T. Brannick, Anson Seers, Robert J. Vandenberg, and Larry J. Williams. "Exploratory and confirmatory factor analysis: Guidelines, issues, and alternatives." Journal of organizational behavior (1997): 667-683.

22. Schreiber, James B., Amaury Nora, Frances K. Stage, Elizabeth A. Barlow, and Jamie King. "Reporting structural equation modeling and confirmatory factor analysis results: A review." The Journal of educational research 99, no. 6 (2006): 323-338.

23. Jackson, Dennis L., J. Arthur Gillaspy Jr, and Rebecca Purc-Stephenson. "Reporting practices in confirmatory factor analysis: an overview and some recommendations." Psychological methods 14, no. 1 (2009): 6.

24. DiStefano, Christine, and Brian Hess. "Using confirmatory factor analysis for construct validation: An empirical review." Journal of Psychoeducational Assessment 23, no. 3 (2005): 225-241.

25. Schmitt, Thomas A. "Current methodological considerations in exploratory and confirmatory factor analysis." Journal of Psychoeducational Assessment 29, no. 4 (2011): 304-321.

26. Fleiss, J. L. "Review papers: The statistical basis of meta-analysis." Statistical methods in medical research 2 , no. 2 (1993): 121-145.

27. Bornstein, Robert F. "Exposure and affect: overview and meta-analysis of research, 1968-1987." Psychological bulletin 106, no. 2 (1989): 265.

28. Eagly, Alice H., Mona G. Makhijani, and Bruce G. Klonsky. "Gender and the evaluation of leaders: A metaanalysis." Psychological bulletin 111, no. 1 (1992): 3.

29. Hillocks Jr, George. "What works in teaching composition: A meta-analysis of experimental treatment studies." American journal of Education 93, no. 1 (1984): 133-170.

30. Raykov, Tenko, and Keith F. Widaman. "Issues in applied structural equation modeling research." Structural Equation Modeling: A Multidisciplinary Journal 2, no. 4 (1995): 289-318.

31. McCown, Robert L., Graeme L. Hammer, John Norman Gresham Hargreaves, Dean P. Holzworth, and David M. Freebairn. "APSIM: a novel software system for model development, model testing and simulation in agricultural systems research." Agricultural systems 50, no. 3 (1996): 255-271.

32. Kriegeskorte, Nikolaus. "Pattern-information analysis: from stimulus decoding to computational-model testing." Neuroimage 56, no. 2 (2011): 411-421.

33. Marcoulides, George A., and Ronald H. Heck. "Organizational culture and performance: Proposing and testing a model." Organization science 4, no. 2 (1993): 209-225.

34. Fraser, Gordon, Franz Wotawa, and Paul E. Ammann. "Testing with model checkers: a survey." Software Testing, Verification and Reliability 19, no. 3 (2009): 215-261.

35. Homer, Jack B. "Partial-model testing as a validation tool for system dynamics (1983)." System Dynamics Review 28 , no. 3 (2012): 281-294

36. Dheenadhayalan, V. "An Analysis of Financial Health of Select Indian Bulk Drugs and Formulations Companies* Mrs. R. Selvi."

37. Dheenadhayalan, V., and R. Devianbarasi. "Financial health of cooperative sugar mills-a case study of NPKRR cooperative sugar mill ltd." Indian Cooperative Review 46, no. 3 (2009): 192-197.

38. Banana, K. R. I. S. H. N. A., and R. V. Chepuri. "Role of recovery channels in managing non-performing assets in scheduled commercial banks." International Journal for Innovative Research in Multidisciplinary Field 2, no. 10 (2016): 355-359.

39. Dheenadhayalan, V. "Mudra-A Tool for Uplifting Micro Enterprises in India." International Journal in Management \& Social Science 4, no. 12 (2016): 235-246.

40. Dheenadhayalan, V., and D. Rajaprabu. "Loan Assets in New Private Sector Banks in India." Asian Journal of Management 5, no. 3 (2014): 347-353.

41. Dheenadhayalan, V. "Mudra-A Tool for Uplifting Micro Enterprises in India." International Journal in Management \& Social Science 4, no. 12 (2016): 235-246.

42. Dheenadhayalan, V. "Impact of E-Commerce on the Changes in Consumer's Buying Behaviour in Malappuram District." Annals of the Romanian Society for Cell Biology (2021): 3441-3452.

43. Dheenadhayalan, V., and R. Shanmuga Priya. "Influencing Factors on Purchase Decision of Women Two-Wheeler Users." Annals of the Romanian Society for Cell Biology (2021): 3430-3440.

44. Dr. Amol Lokhande, Dr. C. Venkateswaran, Dr. M. Ramachandran, C. Vidhya, R. Kurinjimalar, A Study on Various Implications on Reusing in Manufacturing, REST Journal on Emerging trends in Modelling and Manufacturing 7(2), 2021, 63-69. 
45. Dr. Amol Lokhande, Dr. C. Venkateswaran, Dr. M. Ramachandran, S. Chinnasami, T. Vennila, A Review on Various Implications on Re engineering in Manufacturing, REST Journal on Emerging trends in Modelling and Manufacturing Vol: 7(3), 2021,70-75.

46. Dr. Amol Lokhande, Dr. C. Venkateswaran, Dr. M. Ramachandran, C. Sathiyaraj, K. Nathiya, Recycling Process Impact in Current Scenario Manufacturing: A Study, Recent trends in Management and Commerce Vol: 2(1), 2021, 20-25.

47. Gadde Mehar Chaitanya, M.P.Jenarthanan, C. Sathiyaraj, A Review on Glass fibre Reinforced Composites with Different Matrix, REST Journal on Emerging trends in Modelling and Manufacturing Vol: 7(1), 2021, 18-24.

48. R. Kurinjimalar, S. Vimala, M. Silambarasan, S. Chinnasami, A Review on Coir fibre Reinforced Composites with Different Matrix, REST Journal on Emerging trends in Modelling and Manufacturing Vol: 7(2), 2021, 25-32.

49. P. K. Chidambaram, Dr. Amol Lokhande, Dr. M. Ramachandran, Vimala Saravanan, Vidhya Prasanth, A Review on Biodiesel Properties and Fatty acid composites, REST Journal on Emerging trends in Modelling andManufacturing Vol: 7(3), 2021, 87-93.

50. Sharma, Vikrant, B. D. Gidwani, Vikram Sharma, and M. L. Meena. "Implementation model for cellular manufacturing system using AHP and ANP approach." Benchmarking: An International Journal (2019).

51. Sharma, Vikrant, Atul Kumar, and Mukesh Kumar. "A framework based on BWM for big data analytics (BDA) barriers in manufacturing supply chains." Materials Today: Proceedings (2021).

52. Sharma, V., and B. D. Gidwani. "A survey on the status of cellular manufacturing system implementation in Indian manufacturing industries." J. Prod. Res. Manage. 9, no. 3 (2019): 1-8.

53. Pathak, Sudhir Kumar, Kritika Karwasra, Vikrant Sharma, and Vikram Sharma. "Analysis of Barriers to Green Manufacturing Using Hybrid Approach: An Investigatory Case Study on Indian Automotive Industry." Process Integration and Optimization for Sustainability (2021): 1-16.

54. Kumar, Atul, Vikrant Sharma, and N. S. Baruaole. "Experimental investigation of TIG welding of stainless steel 202 and stainless steel 410 using Taguchi technique." Mody University International Journal of Computing and Engineering Research 1, no. 2 (2017): 98-101.

55. Sharma, Vikrant, Sundeep Kumar, and M. L. Meena. "Key criteria influencing cellular manufacturing system: a fuzzy AHP model." Journal of Business Economics (2021): 1-20.

56. Sharma, Vikrant, Vikram Sharma, and Kritika Karwasra. "A decision framework for green manufacturing indicators using fuzzy AHP-ELECTRE I: a case study of the steering system manufacturer." International Journal of Sustainable Engineering 14, no. 6 (2021): 1332-1341.

57. Sharma, Vikrant, B. D. Gidwani, Vikram Sharma, and M. L. Meena. "Modeling the Interactions Among Critical Criteria of a Cellular Manufacturing System." IEEE Engineering Management Review 49, no. 1 (2020): 148-164.

58. Pandey, Vivek, Mukul Kant Paliwal, Vikrant Sharma, and Atul Kumar. "Performance Characteristics of Diesel Engine on Sesame Oil-Diesel Blended Fuel."

59. Kumar, Mukesh, Atul Kumar, Vikrant Sharma, and M. I. T. S. Lakshmangarh. "Risk Analysis of Water Tubes in Boiler using FEMA Risk Analysis of Water Tubes in Boiler using FEMA."

60. Soniya Sriram, M. Ramachandran, Sathiyaraj Chinnasamy, G. Mathivanan. "A Review on Multi-Criteria DecisionMaking and Its Application”. REST Journal on Emerging trends in Modelling and Manufacturing, 7(4), 2021:101107.

61. Sathiyaraj Chinnasamy; M. Ramachandran; Kurinjimalar Ramu; P. Anusuya, "Study on Fuzzy ELECTRE Method with Various Methodologies", REST Journal on Emerging trends in Modelling and Manufacturing, 7(4), 2021: 108115 .

62. S. Chinnasami; M. Ramachandran; P. Vidhya; M. Gowri. "Study of Executive Director for Administrative Services on Moyamoya Disease and Energy application", REST Journal on Emerging trends in Modelling and Manufacturing, 7(4), 2021: 116-124 